\title{
ON THE RANGE OF THE RADON $d$-PLANE TRANSFORM AND ITS DUAL
}

\author{
FULTON B. GONZALEZ
}

\begin{abstract}
We present direct, group-theoretic proofs of the range theorem for the Radon $d$-plane transform $f \rightarrow \hat{f}$ on $\mathscr{S}\left(\mathbb{R}^{n}\right)$. (The original proof, by Richter, involves extensive use of local coordinate calculations on $G(d, n)$, the Grassmann manifold of affine $d$-planes in $\mathbb{R}^{n}$.) We show that moment conditions are not sufficient to describe this range when $d<n-1$, in contrast to the compactly supported case. Finally, we show that the dual $d$-plane transform maps $\mathscr{E}(G(d, n))$ surjectively onto $\mathscr{E}\left(\mathbb{R}^{n}\right)$.
\end{abstract}

\section{INTRODUCTION}

In this article we investigate the $d$-dimensional Radon transform $f \rightarrow \hat{f}$ on $\mathbb{R}^{n}$, where $d<n-1$. This transform integrates functions on $\mathbb{R}^{n}$ over $d$-dimensional planes, and so maps functions on $\mathbb{R}^{n}$ to functions on $G(d, n)$, the affine Grassmann manifold of $d$-dimensional planes in $\mathbb{R}^{n}$. One of the most interesting problems concerning such transforms is how to characterize the range of certain function and distribution spaces on $\mathbb{R}^{n}$, such as $\mathscr{D}\left(\mathbb{R}^{n}\right)$, $\mathscr{S}\left(\mathbb{R}^{n}\right)$, and $\mathscr{E}^{\prime}\left(\mathbb{R}^{n}\right)$.

For $d=n-1$, the ranges of $\mathscr{D}, \mathscr{S}$, and $\mathscr{E}^{\prime}$ are characterized by the Helgason moment conditions [9]. For $d<n-1$, it is still possible to characterize the range of $\mathscr{D}\left(\mathbb{R}^{n}\right)$ by moment conditions [10], but the situation for $\mathscr{S}\left(\mathbb{R}^{n}\right)$ is quite different.

In fact, moment conditions do not suffice to describe the range $\mathscr{S}\left(\mathbb{R}^{n}\right)^{\wedge}$, as will be seen from a simple counterexample in $\S 2$. It turns out that the range $\mathscr{S}\left(\mathbb{R}^{n}\right)^{-}$can be described as the space of rapidly decreasing functions on $G(d, n)$ satisfying a system of second-order partial differential equations. For $d=1$ and $n=3$, this result was already obtained by Fritz John in 1938 [14]. For arbitrary $d$ and $n$, these differential equations were first given explicitly by Gelfand, Gindikin, and Graev [2] in terms of the local coordinates on $G(d, n)$. (See also Gelfand-Graev-Shapiro [1] for the analogous results on $\mathbb{C}^{n}$.) However, their proof omitted many details. In 1984, Grinberg [7]

Received by the editors June 6, 1989.

1980 Mathematics Subject Classification (1985 Revision). Primary 44A05; Secondary 43A85, $53 \mathrm{C} 65,58 \mathrm{C} 35$.

Key words and phrases. Radon $d$-plane transform, range theorem, moment conditions, Euclidean motion group, dual Radon transform, left regular representation. 
described the range $\mathscr{S}\left(\mathbb{R}^{n}\right)^{\wedge}$ in terms of both moment conditions and differential equations. Finally, in 1986, a complete proof characterizing the range $\mathscr{S}\left(\mathbb{R}^{n}\right)^{\wedge}$ in terms of the differential equations of Gelfand, et al. was obtained by Richter [16]. Richter also provided a range characterization in terms of differential equations involving the infinitesimal left regular representation of the Euclidean motion group $E(n)$ on $G(d, n)$. The proof used extensive local coordinate calculations on $G(d, n)$.

In this paper, we present a more direct, group-theoretic proof of the range theorem. As a consequence of a part of the proof, we obtain a proof of the surjectivity of the dual $d$-plane transform on the space $\mathscr{E}\left(\mathbb{R}^{n}\right)$. This was proven by Hertle [13] in the case $d=n-1$.

In $\S 2$, we define the space $\mathscr{S}(G(d, n))$ of rapidly decreasing functions on $G(d, n)$ and the partial Fourier transform on this space. We also prove certain fundamental properties of this partial Fourier transform. Our definition of rapidly decreasing functions on $G(d, n)$ is equivalent to, but quite different in formulation from, that of Richter. However, it can also be easily extended to define the Schwartz functions on a homogeneous unitary $K$-vector bundle, where $K$ is a compact Lie group. In this section, we will also present the counterexample referred to above.

In $\S 3$, we investigate the infinitesimal left regular representation of the Euclidean motion group on $\mathbb{R}^{n}$ and on $G(d, n)$, and its behavior under the Radon transform. We also introduce the differential operators needed in the statement of the range theorem.

In $\S 4$ we prove the range theorem. Finally in $\S 5$, we prove the surjectivity of the dual $d$-plane transform on $\mathscr{E}\left(\mathbb{R}^{n}\right)$. We also present an interesting problem connected with the nullspace of the dual $d$-plane transform.

The author is indebted to Professors S. Helgason and E. T. Quinto for valuable discussions.

\section{RAPIDLY DECREASING FUNCTIONS AND}

THE PARTIAL FOURIER TRANSFORM ON $G(d, n)$

Let $1 \leq d \leq n-1$. The space $G(d, n)$ of $d$-planes in $\mathbb{R}^{n}$ is a homogeneous space of the group $E(n)$ of isometries of $\mathbb{R}^{n}$. It is also a vector bundle over the Grassmann manifold $G_{d, n}$ of $d$-dimensional subspaces of $\mathbb{R}^{n}$, the projection $\pi_{d}$ of $G(d, n)$ onto $G_{d, n}$ being the mapping which associates to any $\xi \in$ $G(d, n)$ the parallel $d$-plane $\sigma$ through the origin. The fiber $\pi_{d}^{-1}(\sigma)$ of $\sigma \in$ $G_{d, n}$ is naturally identified with $\sigma^{\perp} \approx \mathbb{R}^{n-d}$.

If $\sigma^{\perp}$ is an arbitrary fiber and $\varphi \in \mathscr{E}(G(d, n))$, then the restriction of $\varphi$ to $\sigma^{\perp}$ will be denoted $\left.\varphi\right|_{\sigma^{\perp}}$. Define the differential operator $\square$ on $G(d, n)$ by

$$
\left.(\square \varphi)\right|_{\sigma^{\perp}}=\Delta_{\sigma^{\perp}}\left(\left.\varphi\right|_{\sigma^{\perp}}\right)
$$

for all $\varphi \in \mathscr{E}(G(d, n)), \Delta_{\sigma^{\perp}}$ being the Laplacian on $\sigma^{\perp}$. $\square$ is invariant under the action of $E(n)$ on $G(d, n)$ [9]. (See also $\S 4$ below.) 
The $d$-dimensional Radon transform $f \rightarrow \hat{f}$ is defined by

$$
\hat{f}(\xi)=\int_{\xi} f(x) d m(x)
$$

for any function $f$ on $\mathbb{R}^{n}$ integrable on $d$-planes, $d m(x)$ being the Euclidean measure on the $d$-planes $\xi$.

Consider the parametrization of $G(d, n)$ given by

$$
\xi \leftrightarrow(\sigma, x)
$$

where $\sigma=\pi_{d}(\xi)$ and $\{x\}=\xi \cap \sigma^{\perp}$. By [9], the range $\mathscr{D}\left(\mathbb{R}^{n}\right)^{\wedge}$ is the space $\mathscr{D}_{H}(G(d, n))$ consisting of all $\varphi \in \mathscr{D}(G(d, n))$ satisfying the condition that for each $m \in \mathbb{Z}^{+}$, there exists a homogeneous degree $m$ polynomial $P_{m}$ on $\mathbb{R}^{n}$ with

$$
\int_{\sigma^{\perp}} \varphi(\sigma, x)\langle x, u\rangle^{m} d \sigma^{\perp}(x)=P_{m}(u)
$$

for all $u \in \sigma^{\perp}, d \sigma^{\perp}$ being the Euclidean measure on $\sigma^{\perp}$. When $d=n-1$, these moment conditions also characterize the range $\mathscr{S}\left(\mathbb{R}^{n}\right)^{\wedge}$. We also refer the reader to [17], in which the range $L_{c}^{2}\left(\mathbb{R}^{n}\right)^{\wedge}$ is described in terms of the above moment and other intregrability conditions.

A natural question to ask is whether the moment conditions (3) also suffice to describe the range $\mathscr{S}\left(\mathbb{R}^{n}\right)^{\wedge}$ when $d<n-1$.

Now it is clear that the range $\mathscr{S}\left(\mathbb{R}^{n}\right)^{\wedge}$ consists of functions on $G(d, n)$ which decrease rapidly on its fibers. To make this precise, we first need to formulate a definition of the space $\mathscr{S}(G(d, n))$ of rapidly decreasing functions on the manifold $G(d, n)$.

It is difficult to define $\mathscr{S}(G(d, n))$ by means of the parametrization (2), since $x$ and $\sigma$ are not independent parameters $\left(x \in \sigma^{\perp}\right)$. Instead we use the fact that $G(d, n)$ is a homogeneous $O(n)$-unitary vector bundle [19].

Let $e_{1}, e_{2}, \ldots, e_{n}$ be the usual basis of $\mathbb{R}^{n}$, and let $\sigma_{0}$ denote the subspace $\mathbb{R} e_{1}+\mathbb{R} e_{2}+\cdots+\mathbb{R} e_{d}$. We identify $\sigma_{0}^{\perp}$ with $\mathbb{R}^{n-d}=\mathbb{R} e_{d+1}+\cdots+\mathbb{R} e_{n}$. Let $\pi: k \mapsto k \cdot \sigma_{0}$ be the canonical projection of $O(n)$ onto $G_{d, n}$. A compact subset $M \subset G_{d, n}$ is called full if $M$ is the closure of its interior. If $M$ is a full compact subset of $G_{d, n}$, we say that it admits a local cross section into $O(n)$ if $M$ is contained in an open set $V \subset G_{d, n}$ such that there exists a local $C^{\infty}$ cross section $\eta$ of $V$ into a submanifold of $O(n)$ (i.e., $\pi \circ \eta$ is the identity map on $V$ ). Note that $G_{d, n}$ is a finite union of full compact sets which admit local cross sections.

By definition, the function $\varphi$ belongs to $\mathscr{S}(G(d, n))$ if and only if $\varphi \in$ $\mathscr{E}(G(d, n))$ and satisfies the estimate

$$
\sup _{\substack{\sigma \in M \\ x \in R^{n-d}}}(1+\|x\|)^{r}\left|E_{\sigma} D_{x} \varphi(\sigma, \eta(\sigma) \cdot x)\right|<\infty
$$


for all $r \in \mathbb{Z}^{+}$, for all differential operators $E$ on $G_{d, n}$, for all constant coefficient differential operators $D$ on $\mathbb{R}^{n-d}$, and for all full compact subsets $M$ of $G_{d, n}$ admitting local cross sections $\eta$ into $O(n)$. Note that $\eta(\sigma) \cdot\left(\sigma_{0}, x\right)=$ $(\sigma, \eta(\sigma) \cdot x)$. In the estimate (4), we could also have replaced $D$ by a power of the operator $\square$. Note also that a local trivialization of the vector bundle $G(d, n)$ is given by

$$
\begin{aligned}
\Gamma: V \times \mathbb{R}^{n-d} & \rightarrow \pi_{d}^{-1}(V), \\
(\sigma, x) & \mapsto(\sigma, \eta(\sigma) \cdot x)
\end{aligned}
$$

for all $x \in \mathbb{R}^{n-d}, \sigma \in V$, where $V \subset G_{d, n}$ is an open set admitting a local cross section into $O(n)$.

The advantage in using local cross sections to parametrize $G(d, n)$ locally, as in (5), is that the distance from the origin to the $d$-plane $(\sigma, \eta(\sigma) \cdot x)$ is $\|x\|$. Moreover, the transition matrices between adjacent local trivial bundles are all orthogonal. The definition (4) may also be used to define the rapidly decreasing functions on a homogeneous unitary $K$-vector bundle, where $K$ is a compact Lie group [19].

Now let $\varphi \in \mathscr{S}(G(d, n))$. The partial Fourier transform of $\varphi$ is the function $\tilde{\varphi}$ on $G(d, n)$ given by

$$
\tilde{\varphi}(\sigma, u)=\int_{\sigma^{\perp}} \varphi(\sigma, x) e^{-i\langle x, u\rangle} d \sigma^{\perp}(x), \quad u \in \sigma^{\perp} .
$$

Clearly, $\tilde{\varphi} \in \mathscr{E}(G(d, n))$. The inverse partial Fourier transform is defined likewise.

Proposition 2.1. If $\varphi \in \mathscr{S}(G(d, n))$, then $\tilde{\varphi} \in \mathscr{S}(G(d, n))$.

The proof proceeds as in the Euclidean case and will be omitted. by

Next, for each function $f \in \mathscr{S}\left(\mathbb{R}^{n}\right)$, we define the function $\varphi_{f}$ on $G(d, n)$

$$
\varphi_{f}(\sigma, x)=f(x), \quad \sigma \in G_{d, n}, x \in \sigma^{\perp} .
$$

Clearly, $\varphi_{f} \in \mathscr{E}(G(d, n))$.

Lemma 2.2. $\varphi_{f} \in \mathscr{S}(G(d, n))$.

The proof is tedious but straightforward. For details, see [3].

Proposition 2.3. If $f \in \mathscr{S}\left(\mathbb{R}^{n}\right)$, then $\hat{f} \in \mathscr{S}(G(d, n))$.

Proof. The Fourier transform $\tilde{f}$ belongs to $\mathscr{S}\left(\mathbb{R}^{n}\right)$, so by Lemma 2.2, $\varphi_{\tilde{f}} \in$ $\mathscr{S}(G(d, n))$. According to the Projection-slice Theorem [10], $\varphi_{\tilde{\varphi}}$ is the partial Fourier transform of $\hat{f}$. Taking inverse partial Fourier transform, Proposition 2.1 implies that $\hat{f} \in \mathscr{S}(G(d, n))$.

With the definition of $\mathscr{D}_{H}(G(d, n))$ in mind, we define $\mathscr{S}_{H}(G(d, n))$ to be the space of all $\varphi \in \mathscr{S}(G(d, n))$ satisfying the moment conditions (3). Clearly, the Radon transform $f \mapsto \hat{f}$ is a 1-1 map of $\mathscr{S}\left(\mathbb{R}^{n}\right)$ into $\mathscr{S}_{H}(G(d, n))$ and 
for $d=n-1$, it is a bijection [10]. On the other hand, it is not onto for $d<n-1$. In fact, we produce below a function $\varphi \in \mathscr{S}_{H}(G(d, n))$ which does not belong to the range $\mathscr{S}\left(\mathbb{R}^{n}\right)^{\wedge}$.

If $\sigma \in G_{d, n}$, any $u \in \sigma^{\perp}$ may be considered as a vector field acting on $\sigma^{\perp}$. This vector field will still be denoted by $u$. For $\varphi \in \mathscr{S}_{H}(G(d, n))$, the moment conditions translate to the following differentiability conditions for the partial Fourier transform at the origin of each $\sigma^{\perp}$ :

$$
\left(\left.u^{m} \tilde{\varphi}\right|_{\sigma^{\perp}}\right)(0)=P_{m}(u), \quad u \in \sigma^{\perp} .
$$

This is a condition that holds only at the origin in each $\sigma^{\perp}$. Consequently, one can find a function $\psi \in \mathscr{D}(G(d, n))$ satisfying the following two conditions:

(i) For some $x_{0} \in \mathbb{R}^{n}$, there exist $\sigma_{0}, \sigma_{1} \in G_{d, n}$ with $x_{0} \in \sigma_{0}^{\perp}, x_{0} \in \sigma_{1}^{\perp}$ such that $\psi\left(\sigma_{0}, x_{0}\right) \neq \psi\left(\sigma_{1}, x_{0}\right)$.

(ii) $\psi(\sigma, x)=0$ for all $\sigma \in G_{d, n}$ and all $x \in \sigma^{\perp}$ such that $\|x\| \leq 1$. Now by Proposition 2.1, $\psi=\tilde{\varphi}$ for some $\varphi \in \mathscr{S}(G(d, n))$. By (ii), $\varphi \in$ $\mathscr{S}_{H}(G(d, n))$ with each polynomial $P_{m}$ identically zero. On the other hand, (i) implies that $\varphi \notin \mathscr{S}\left(\mathbb{R}^{n}\right)^{-}$, because if $\varphi=\hat{f}$, then $\psi\left(\sigma_{0}, x_{0}\right)=\tilde{f}\left(x_{0}\right)=$ $\psi\left(\sigma_{1}, x_{0}\right)$.

We remark that for $d=n-1$, condition (i) above cannot be fulfilled. We also remark that if $\varphi \in \mathscr{D}_{H}(G(d, n))$, its partial Fourier transform $\tilde{\varphi}$ is analytic on each fiber $\sigma^{\perp}$, and so is determined by its derivatives at the origin. Hence the global behavior of $\tilde{\varphi}$ is determined by the moment conditions on $\varphi$. This explains the difference between the results for $\mathscr{D}\left(\mathbb{R}^{n}\right)^{\wedge}$ and $\mathscr{S}\left(\mathbb{R}^{n}\right)^{\wedge}$.

\section{The INFINITESIMAL ACTION OF THE EUCLIDEAN MOTION GROUP}

In what follows we assume $d<n-1$. In order to facilitate later calculations we also adopt the following conventions. Suppose $M$ is a manifold of dimension $m$. Let $f$ be a function, $D$ a differential operator, and $T$ a distribution on $M$. If $\tau: M \rightarrow M$ is a diffeomorphism, $f^{\tau}$ will denote the function $f \circ \tau^{-1}, D^{\tau}$ the differential operator $f \rightarrow(D(f \circ \tau)) \circ \tau^{-1}$, and $T^{\tau}$ the distribution $f \rightarrow T(f \circ \tau)$. Then $(D f)^{\tau}=D^{\tau} f^{\tau}$, and if $D^{\prime}$ is another differential operator, $\left(D D^{\prime}\right)^{\tau}=D^{\tau} D^{\prime \tau}$. If $\tau$ preserves a nonvanishing $m$-form $\omega$ on $M$ (so the adjoint $D^{*}$ of each differential operator $D$ is defined), then $\left(D^{*}\right)^{\tau}=\left(D^{\tau}\right)^{*}$ and $(D T)^{\tau}=D^{\tau} T^{\tau}$.

The Euclidean motion group $E(n)=O(n) \times \mathbb{R}^{n}$ is the set of all ordered pairs $(k, v)$ where $k \in O(n)$ and $v \in \mathbb{R}^{n}$, with group law $(k, v) \cdot\left(k^{\prime}, v^{\prime}\right)$ $=\left(k k^{\prime}, k \cdot v^{\prime}+v\right)$. We have $\mathbb{R}^{n}=E(n) / O(n)$ and $G(d, n)=E(n) / E(d) \times$ $O(n-d)$. The left action of $E(n)$ on $\mathbb{R}^{n}$ and $G(d, n)$ will both be denoted by $\tau$. If $k \in O(n)$, we will, as we have been previously doing, replace $\tau(k) x$ by the simpler expression $k \cdot x$.

$E(n)$ may also be identified with the subgroup of $\mathrm{GL}(n+1, \mathbb{R})$ consisting of the matrices $\left(\begin{array}{ll}k & v \\ 0 & 1\end{array}\right)$, where $k \in O(n)$ and $v \in \mathbb{R}^{n}$. The left regular representation of $E(n)$ on $\mathscr{E}\left(\mathbb{R}^{n}\right)$ and $\mathscr{E}(G(d, n))$ will be denoted by $\lambda$ and 
$\nu$, respectively: if $g \in E(n)$, then $\lambda(g) f=f^{\tau(g)}$ and $\nu(g) \varphi=\varphi^{\tau(g)}$ for $f \in \mathscr{E}\left(\mathbb{R}^{n}\right)$ and $\varphi \in \mathscr{E}(G(d, n))$.

Let $\mathfrak{e}$ be the Lie algebra of $E(n)$. Then the infinitesimal left regular representations $d \lambda$ and $d \nu$ of $\mathfrak{e}$ will be extended to the universal enveloping algebra $\mathfrak{U}(\mathfrak{e})$. Explicitly, if $X_{1}, \ldots, X_{r} \in \mathfrak{e}$, then

(6) $\left(d \lambda\left(X_{1} \cdots X_{r}\right) f\right)(x)=\left\{\frac{\partial^{r}}{\partial t_{1} \cdots \partial t_{r}} f\left(\tau\left(\exp \left(-t_{r} X_{r}\right) \cdots \tau\left(-t_{1} X_{1}\right)\right) \cdot x\right)\right\}_{t_{i}=0}$

for all $f \in \mathscr{E}\left(\mathbb{R}^{n}\right)$. A similar expression can be written for $d \nu$.

If $f \in \mathscr{S}\left(\mathbb{R}^{n}\right)$ and $g \in E(n)$, it is immediate that $(\lambda(g) f)^{\wedge}=\nu(g) \hat{f}$. By differentiating inside the integral sign we have

$$
(d \lambda(U) f)^{\wedge}=d \nu(U) \hat{f}
$$

for all $U \in \mathfrak{U}(\mathfrak{e})$. (By (8) below, $d \lambda(U) f \in \mathscr{S}$ for all $U \in \mathfrak{U}(\mathfrak{e})$, so differentiation inside the integral sign is indeed permissible.)

We may identify the Lie algebra $\mathfrak{e}$ with the Lie subalgebra of $g l(n+1, \mathbb{R})$ consisting of the matrices $\left(\begin{array}{ll}T & v \\ 0 & 0\end{array}\right)$, where $T \in \operatorname{so}(n), v \in \mathbb{R}^{n}$. Let $E_{i j}$ denote the $(n+1) \times(n+1)$ matrix $\left(\delta_{i r} \delta_{s j}\right)_{r, s=1}^{n}$. Write

$$
\begin{aligned}
X_{i j}=E_{i j}-E_{j i}, & 1 \leq i, j \leq n, \\
E_{k}=E_{k, n+1}, & 1 \leq k \leq n+1 .
\end{aligned}
$$

Note that $X_{i i}=0$. e has basis $X_{i j}(i<j)$ and $E_{k} \quad(1 \leq k \leq n)$.

If $U \in \mathfrak{U}(\mathfrak{e}), d \lambda(U)$ and $d \nu(U)$ are differential operators on $\mathbb{R}^{n}$ and $G(d, n)$, respectively. In particular,

$$
d \lambda\left(X_{i j}\right)=x_{i} \frac{\partial}{\partial x_{j}}-x_{j} \frac{\partial}{\partial x_{i}}, \quad d \lambda\left(E_{k}\right)=-\frac{\partial}{\partial x_{k}} .
$$

For $1 \leq i, j, l \leq n$, let $V_{i j l}=E_{i} X_{j l}+E_{j} X_{l i}+E_{l} X_{i j} \in \mathfrak{U}(\mathfrak{e})$. (If any of the indices $i, j, l$ coincide, $V_{i j l}=0$.) By (7),

$$
d \nu\left(V_{i j l}\right) \hat{f}=0
$$

for all $f \in \mathscr{S}\left(\mathbb{R}^{n}\right)$.

Following Richter [16], we define $\mathscr{S}_{D}(G(d, n))$ to be the space of all $\varphi \in$ $\mathscr{S}(G(d, n))$ satisfying the differential equations $d \nu\left(V_{i j l}\right) \varphi=0$. By (9), $\mathscr{S}\left(\mathbb{R}^{n}\right)^{\wedge} \subset \mathscr{S}_{D}(G(d, n))$.

\section{The RANGe OF THE $d$-PLANE TRANSForm}

In this section we show that $\mathscr{S}\left(\mathbb{R}^{n}\right)^{\wedge}=\mathscr{S}_{D}(G(d, n))$. First we require a lemma about the adjoint action of $E(n)$ on the elements $V_{i j l} \in \mathfrak{U}(\mathfrak{e})$. 
Lemma 4.1. Let $k=\left(k_{r s}\right)_{r, s=1}^{n} \in O(n)$ and $v \in \mathbb{R}^{n}$. Suppose $1 \leq i, j, l \leq n$. Then

$$
\begin{aligned}
& \operatorname{Ad}(k) V_{i j l}=\sum_{u<s<r} \operatorname{det}\left[\begin{array}{lll}
k_{u i} & k_{u j} & k_{u l} \\
k_{s i} & k_{s j} & k_{s l} \\
k_{r i} & k_{r j} & k_{r l}
\end{array}\right] \cdot V_{u s r}, \\
& \operatorname{Ad}(v) V_{i j l}=V_{i j l} .
\end{aligned}
$$

Proof. If we consider $E(n)$ as a matrix group, then the adjoint representation is just given by conjugation: $\operatorname{Ad}(g) X=g X g^{-1}(g \in E(n), X \in \mathfrak{e})$. Thus, by a routine computation,

$$
\operatorname{Ad}(k) E_{i}=\sum_{j=1}^{n} k_{j i} E_{j}, \quad \operatorname{Ad}(k) X_{j l}=\sum_{i, r=1}^{n} k_{i j} k_{r l} X_{i r} .
$$

Hence

$$
\begin{aligned}
\operatorname{Ad}(k) V_{i j l} & =\sum_{u, s, r} k_{u i} k_{s j} k_{r l}\left(E_{u} X_{s r}+E_{s} X_{r u}+E_{r} X_{u s}\right) \\
& =\sum_{u, s, r} k_{u i} k_{s j} k_{r l} V_{u s r} .
\end{aligned}
$$

For each fixed $u<s<r$ in the above sum, we have $V_{u r s}=-V_{u s r}$, etc., proving (i). For (ii), write $v=\sum_{r=1}^{n} v_{r} E_{r}$. Then $\operatorname{Ad}(v) X_{j l}=X_{j l}+v_{j} E_{l}-v_{l} E_{j}$ and $\operatorname{Ad}(v) E_{i}=E_{i}$ for all $i, j, l$. Hence

$$
\begin{aligned}
\operatorname{Ad}(v) V_{i j l}= & E_{i}\left(X_{j l}+v_{j} E_{l}-v_{l} E_{j}\right)+E_{j}\left(X_{l i}+v_{l} E_{i}-v_{i} E_{l}\right) \\
& +E_{l}\left(X_{i j}+v_{i} E_{j}-v_{j} E_{i}\right)=V_{i j l} .
\end{aligned}
$$

The next lemma describes how $\mathfrak{U}(\mathfrak{e})$ behaves under the partial Fourier transform.

Lemma 4.2. Let $T \in \operatorname{so}(n)$ and $v \in \mathbb{R}^{n}$. If $\varphi \in \mathscr{S}(G(d, n))$, then

(i) $(d \nu(T) \varphi)^{\sim}=d \nu(T) \tilde{\varphi}$, and

(ii) $(d \nu(v) \varphi)^{\sim}(\sigma, x)=-i\langle v, x\rangle \tilde{\varphi}(\sigma, x)$.

Proof. An easy computation shows that

$$
(\nu(k) \varphi)^{\sim}=\nu(k) \tilde{\varphi}
$$

for all $k \in O(n)$. Differentiating, we obtain (i). For (ii), we note that $\exp (t v)=$ $t v$ so that

$$
\begin{aligned}
(\nu(t v) \varphi)^{\sim}(\sigma, x) & =\int_{\sigma^{\perp}} \varphi\left(\sigma, u-P_{\sigma^{\perp}}(t v)\right) e^{-i\langle u, x\rangle} d \sigma^{\perp}(u) \\
& =e^{-i\left\langle P_{\sigma^{\perp}}(t v), x\right\rangle} \tilde{\varphi}(\sigma, x)=e^{-i t\langle v, x\rangle} \tilde{\varphi}(\sigma, x) .
\end{aligned}
$$

Here $P_{\sigma^{\perp}}(t v)$ is the orthogonal projection of $t v$ on $\sigma^{\perp}$. Differentiating both sides of (11) proves (ii). 
By Proposition 2.1 and Lemma 4.2, $\psi \in \mathscr{S}_{D}(G(d, n))^{\sim}$ if and only if

$$
\left(x_{i} d \nu\left(X_{j l}\right)+x_{j} d \nu\left(X_{l i}\right)+x_{l} d \nu\left(X_{i j}\right)\right) \psi(\sigma, x)=0
$$

for all $i, j, l$. Let $\Lambda_{i j l}$ be the differential operator in (12). Since $d \nu(\operatorname{Ad}(g) U)$ $=d \nu(U)^{\tau(g)}$ for all $g \in E(n), U \in \mathfrak{U}(\mathfrak{e})$, we have by Lemma 4.1

$$
\Lambda_{i j l}^{\tau(k)}=\sum_{u<s<r} \operatorname{det}\left[\begin{array}{ccc}
k_{u i} & k_{u j} & k_{u l} \\
k_{s i} & k_{s j} & k_{s l} \\
k_{r i} & k_{r j} & k_{r l}
\end{array}\right] \Lambda_{u s r} .
$$

Equation (13) may also be obtained by direct computation.

The next three lemmas, due to Richter [16], show that the elements of $\mathscr{S}_{D}(G(d, n))^{\sim}$ are of the form $\varphi_{F}$, where $F \in \mathscr{S}\left(\mathbb{R}^{n}\right)$. Our proofs are based on(13) and are essentially coordinate-free.

Lemma 4.3. Let $\psi \in \mathscr{S}_{D}(G(d, n))^{\sim}$. Then there exists $F \in \mathscr{E}\left(\mathbb{R}^{n} \backslash\{0\}\right) \cap C\left(\mathbb{R}^{n}\right)$ such that $\psi(\sigma, x)=F(x)$ for all $\sigma \in G_{d, n}, x \in \sigma^{\perp}$.

Proof. Fix $x \in \mathbb{R}^{n}$. Define $O\left(x^{\perp}\right)$ to be the subgroup of $O(n)$ consisting of all $k$ such that $k \cdot x=x$. (Note $O\left(0^{\perp}\right)=O(n)$.) Let $s o\left(x^{\perp}\right)$ denote its Lie algebra. $s o\left(x^{\perp}\right)$ consists of all infinitesimal rotations fixing $x$. Assume first $x \neq 0$. We will prove below that for any $Z \in \operatorname{so}\left(x^{\perp}\right)$,

$$
(d \nu(Z) \psi)(\sigma, x)=0
$$

for all $\sigma \in G_{d, n}$ such that $x \in \sigma^{\perp}$. It will then follow from (14) that

$$
\psi(\sigma, x)=\psi\left(\sigma^{\prime}, x\right)
$$

for all $\sigma, \sigma^{\prime} \in G_{d, n}$ such that $x \in \sigma^{\perp} \cap \sigma^{\perp}$. Thus $\psi$ does not depend on the argument $\sigma$ and (15) can be used to define the function $F$ away from the origin. It is easily seen that $F \in \mathscr{E}\left(\mathbb{R}^{n} \backslash\{0\}\right)$. Also since $\operatorname{so}\left((t x)^{\perp}\right)=\operatorname{so}\left(x^{\perp}\right)$ for all $t \neq 0,(14)$ implies that

$$
(d \nu(Z) \psi)(\sigma, t x)=0
$$

for all $Z \in \operatorname{so}\left(x^{\perp}\right), t \neq 0$. Letting $t \rightarrow 0$, we obtain

$$
(d \nu(Z) \psi)(\sigma, 0)=0
$$

for all $Z \in \operatorname{so}\left(x^{\perp}\right)$. Since $x$ is an arbitrary point in $\mathbb{R}^{n} \backslash\{0\},(16)$ holds for all $Z \in \sum_{x \in R^{n}-0} \operatorname{so}\left(x^{\perp}\right)=s o(n)$. Hence (14) holds even when $x=0$, so it can be used to define $F(0)$. It is easy to see that $F$ is continuous at 0 .

We now prove (14). Let $r=\|x\|$, and let $\sigma_{0}$ be the $d$-dimensional subspace spanned by $e_{1}, \ldots, e_{d}$. Since $O(n)$ is transitive on the set of $d$-planes at a given distance from 0 , there exists $k \in O(n)$ such that $k \cdot r e_{n}=x$ and $k \cdot \sigma_{0}=\sigma$. Note that $O\left(x^{\perp}\right)=k\left(\begin{array}{cc}O(n-1) & 0 \\ 0 & 1\end{array}\right) k^{-1}$. Thus, since $Z \in \operatorname{so}\left(x^{\perp}\right)$,

$$
\operatorname{Ad}\left(k^{-1}\right) Z=\sum_{1 \leq i<j<n} c_{i j}(k) X_{i j} \in\left(\begin{array}{cc}
s o(n-1) & 0 \\
0 & 0
\end{array}\right) .
$$


Hence

$$
\begin{aligned}
(d \nu(Z) \psi)(\sigma, x) & =(d \nu(Z) \psi)\left(k \cdot\left(\sigma_{0}, r e_{n}\right)\right) \\
& =(d \nu(Z) \psi)^{\tau\left(k^{-1}\right)}\left(\sigma_{0}, r e_{n}\right) \\
& =\left(d \nu\left(\operatorname{Ad}\left(k^{-1}\right) Z\right) \psi^{\tau\left(k^{-1}\right)}\right)\left(\sigma_{0}, r e_{n}\right) \\
& =\sum_{1 \leq i<j<n} c_{i j}(k)\left(d \nu\left(X_{i j}\right) \psi^{\tau\left(k^{-1}\right)}\right)\left(\sigma_{0}, r e_{n}\right) .
\end{aligned}
$$

To prove (14) it will suffice to prove that each of the above summands is zero. But

$$
\begin{aligned}
\left(r d \nu\left(X_{i j}\right) \psi^{\tau\left(k^{-1}\right)}\right)\left(\sigma_{0}, r e_{n}\right) & =\left(\Lambda_{i j n} \psi^{\tau\left(k^{-1}\right)}\right)\left(\sigma_{0}, r e_{n}\right) \\
& =\left(\Lambda_{i j n}^{\tau(k)} \psi\right)^{\tau\left(k^{-1}\right)}\left(\sigma_{0}, r e_{n}\right) \\
& =\left(\Lambda_{i j n}^{\tau(k)} \psi\right)(\sigma, x) .
\end{aligned}
$$

By (13), $\Lambda_{i j n}^{\tau(k)}$ is a linear combination of operators of the form $\Lambda_{u s r}$. Since $\psi \in \widetilde{\mathscr{S}}_{D}$, the last expression above equals zero. This proves (14) and the lemma.

The next lemma shows that $F$ is also differentiable at the origin.

Lemma 4.4. Let $\psi \in \mathscr{E}(G(d, n))$ satisfy the condition $\psi(\sigma, x)=\psi\left(\sigma^{\prime}, x\right)$ for all $x \in \mathbb{R}^{n}$, and $\sigma, \sigma^{\prime} \in G_{d, n}$ with $x \in \sigma^{\perp} \cap \sigma^{\perp}$. Define the function $F$ on $\mathbb{R}^{n}$ by $F(x)=\psi(\sigma, x)$ for all $x \in \sigma^{\perp}$. Then $F \in \mathscr{E}\left(\mathbb{R}^{n}\right)$.

Proof. As in the preceding lemma, $F \in \mathscr{E}\left(\mathbb{R}^{n} \backslash\{0\}\right) \cap C\left(\mathbb{R}^{n}\right)$. In particular, $F$ is continuous at the origin. We intend to show that for each $i=1, \ldots, n$, there exists a function $\Psi_{i} \in \mathscr{E}(G(d, n))$ such that

$$
\frac{\partial F}{\partial x_{i}}(x)=\Psi_{i}(\sigma, x)
$$

for all $x \neq 0$ in $\sigma^{\perp}$ and all $\sigma \in G_{d, n}$. Since $\Psi_{i}$ satisfies the hypothesis of the present lemma, $\partial F / x_{i}$ can be extended continuously to the origin. An elementary induction then proves that all partial derivatives of $F$ can be continuously extended to the origin.

The proof of (17) consists of several steps.

(A) We first remark that $\psi$ satisfies the relation $\Lambda_{i j l} \psi=0$. In fact, using the notation in the proof of Lemma 4.3, we have

$$
\begin{aligned}
\left(\Lambda_{i j l} \psi\right)(\sigma, x) & =\left(\Lambda_{i j l} \psi\right)^{\tau\left(k^{-1}\right)}\left(\sigma_{0}, r e_{n}\right) \\
& =\left(\Lambda_{i j l}^{\tau\left(k^{-1}\right)} \psi^{\left(\tau\left(k^{-1}\right)\right.}\right)\left(\sigma_{0}, r e_{n}\right) \\
& =\sum_{u<s<n} c_{u s n}(k)\left(\Lambda_{u s n} \psi^{\tau\left(k^{-1}\right)}\right)\left(\sigma_{0}, r e_{n}\right) \\
& =\sum_{u<s<n} c_{u s n}(k) r\left(d \nu\left(X_{u s}\right) \psi^{\tau\left(k^{-1}\right)}\right)\left(\sigma_{0}, r e_{n}\right) .
\end{aligned}
$$


Here $c_{u s n}(k)$ is an appropriate $3 \times 3$ determinant. Now $\psi^{\tau\left(k^{-1}\right)}$ satisfies the hypothesis of the lemma, and $X_{u s} \in\left(\begin{array}{cc}s o(n-1) & 0 \\ 0 & 0\end{array}\right)$, so $\left(d \nu\left(X_{u s}\right) \psi^{\tau\left(k^{-1}\right)}\right)\left(\sigma_{0}, r e_{n}\right)=$ 0 .

(B) Next we examine the relation between $\partial F / \partial x_{i}$ and the corresponding derivatives of $\psi$. Let $\sigma \in G_{d, n}$ and let $u_{1}, \ldots, u_{d}$ be an orthonormal basis of $\sigma$. We may assume that each $u_{j}$ is a column vector with components $u_{i j}$ $(1 \leq i \leq n)$. Recall that we identify each vector $v \in \sigma^{\perp}$ with the corresponding vector field in $\sigma^{\perp}$. We have

$$
\begin{aligned}
\left(d \nu\left(e_{i}\right) \psi\right)(\sigma, x) & =-P_{\sigma^{\perp}}\left(e_{i}\right) \psi(\sigma, x)=-\left\langle P_{\sigma^{\perp}}\left(e_{i}\right), \operatorname{grad} F(x)\right\rangle \\
& =-\left\langle e_{i}, P_{\sigma^{\perp}}(\operatorname{grad} F(x))\right\rangle \\
& =-\left\langle e_{i}, \operatorname{grad} F(x)-\sum_{j=1}^{d}\left\langle\operatorname{grad} F(x), u_{j}\right\rangle u_{j}\right\rangle \\
& =-\frac{\partial F}{x_{i}}+\sum_{j=1}^{d} \sum_{r=1}^{n} \frac{\partial F}{\partial x_{r}} u_{r j} u_{i j} \\
& =-\frac{\partial F}{x_{i}}\left(1-\sum_{j=1}^{d} u_{i j}^{2}\right)+\sum_{j=1}^{d} u_{i j} \sum_{\substack{r=1 \\
r \neq i}} \frac{\partial F}{x_{r}} u_{r j}
\end{aligned}
$$

Now if $X \in \operatorname{so}(n), F(\exp (t X) \cdot x)=\psi(\exp (t X) \cdot \sigma, \exp (t X) \cdot x)$. Thus

$$
(d \lambda(X) F)(x)=(d \nu(X) \psi)(\sigma, x)
$$

for all $X \in \operatorname{so}(n)$. Hence by part (A) and (12),

$$
\begin{aligned}
& x_{i}\left(d \nu\left(X_{r j}\right)\right) \psi(\sigma, x)+x_{r}\left(d \nu\left(X_{j i}\right) \psi\right)(\sigma, x) \\
& \quad=-x_{j}\left(d \lambda\left(X_{i r}\right) F\right)(x)=-x_{j}\left(x_{i} \frac{\partial F}{\partial x_{r}}-x_{r} \frac{\partial F}{\partial x_{i}}\right)(x) .
\end{aligned}
$$

Therefore,

$$
\frac{\partial F}{\partial x_{r}}=\frac{1}{x_{i} x_{j}}\left(x_{j} x_{r} \frac{\partial F}{\partial x_{i}}-x_{i} d \nu\left(X_{r j}\right) \psi-x_{r} d \nu\left(X_{j i}\right) \psi\right)
$$

In (20), the derivatives of $F$ are understood as being evaluated at $x \in \sigma^{\perp}$, and the derivatives of $\psi$ at $(\sigma, x)$. Substituting (20) into (18), and then using 
(12), we have

$$
\begin{aligned}
\left(d \nu\left(e_{i}\right) \psi\right)(\sigma, x)= & -\frac{\partial F}{\partial x_{i}}\left(1-\sum_{j=1}^{d} u_{i j}^{2}-\sum_{\substack{r=1 \\
r \neq i}} \sum_{j=1}^{d} \frac{u_{i j} u_{r j} x_{r}}{x_{i}}\right) \\
& -\sum_{\substack{r=1 \\
r \neq i}} \sum_{j=1}^{d}\left(\frac{u_{i j} u_{r j}}{x_{j}} d \nu\left(X_{r j}\right) \psi+\frac{u_{i j} u_{r j} x_{r}}{x_{i} x_{j}} d \nu\left(X_{j i}\right) \psi\right) \\
= & -\frac{\partial F}{\partial x_{i}}\left(1-\sum_{j=1}^{d} u_{i j}^{2}-\sum_{j=1}^{d} \frac{u_{i j}}{x_{i}} \sum_{\substack{r=1 \\
r \neq i}}^{n} u_{r j} x_{r}\right) \\
& -\sum_{\substack{r=1 \\
r \neq i}}^{n} \sum_{j=1}^{d} \frac{u_{i j} u_{r j}}{x_{i} x_{j}}\left(x_{i} d \nu\left(X_{r j}\right)+x_{r} d \nu\left(X_{j i}\right)\right) \psi \\
= & -\frac{\partial F}{\partial x_{i}}\left(1-\sum_{j=1}^{d} u_{i j}^{2}-\sum_{j=1}^{d} \frac{u_{i j}}{x_{i}}\left(-u_{i j} x_{i}\right)\right) \\
& +\sum_{r=1}^{n} \sum_{j=1}^{d} \frac{u_{i j} u_{r j} d \nu\left(X_{i r}\right) \psi}{x_{i}}=-\frac{\partial F}{\partial x_{i}}+\frac{1}{x_{i}} \sum_{r=1}^{n} A_{i r}(\sigma) d \nu\left(X_{i r}\right) \psi \\
= & \\
&
\end{aligned}
$$

Here $A_{i r}(\sigma)=\sum_{j=1}^{d} u_{i j} u_{r j}$ depends only on $\sigma \in G_{d, n}$. By (21) we have

$$
\frac{\partial F}{\partial x_{i}}=-\nu\left(e_{i}\right) \psi+\frac{1}{x_{i}} \sum_{r=1}^{n} A_{i r}(\sigma) d \nu\left(X_{i r}\right) \psi
$$

(C) Let $\Psi_{i}$ be the right-hand side of (22). To show that $\Psi_{i} \in \mathscr{E}(G(d, n))$, it suffices to show that the second expression on the right-hand side of $(22)$ equals a $C^{\infty}$ function $\Phi_{i}$ on $G(d, n)$. This will prove the lemma. In order to prove this claim, we first assert that

$$
x_{m} \sum_{r=1}^{n} A_{i r}(\sigma) d \nu\left(X_{i r}\right) \psi=x_{i} \sum_{r=1}^{n} A_{i r}(\sigma) d \nu\left(X_{m r}\right) \psi
$$

for all $1 \leq i, m \leq n$. For this it suffices to prove that for each $1 \leq j \leq d$

$$
x_{m} \sum_{r=1}^{n} u_{r j} d \nu\left(X_{i r}\right) \psi=x_{i} \sum_{r=1}^{n} u_{r j} d \nu\left(X_{m r}\right) \psi .
$$

For then, each side of (24) can be multiplied by $u_{i j}$ and summed over $j$. Now to prove (24), it may be assumed that $i=1$ and $m=2$. The proof for the 
other cases is analogous. By (12) we have the following system of $n$ equations.

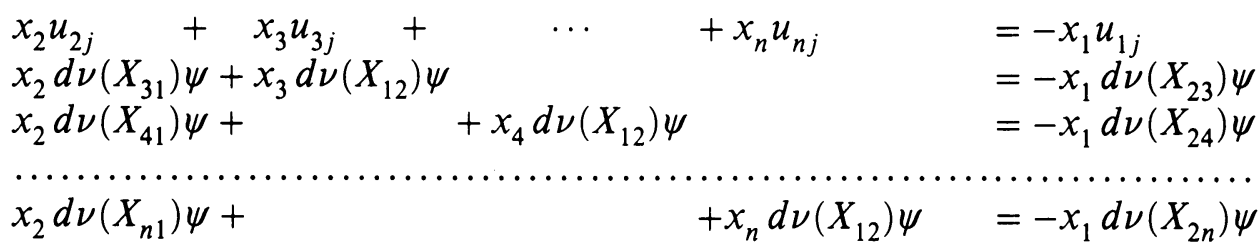

Eliminating the variables $x_{3}, \ldots, x_{n}$, we obtain

$$
\begin{aligned}
& x_{2}\left(u_{2 j} d \nu\left(X_{12}\right) \psi-u_{3 j} d \nu\left(X_{31}\right) \psi-u_{4 j} d \nu\left(X_{41}\right) \psi-\cdots-u_{n j} d \nu\left(X_{n 1}\right) \psi\right. \\
& \quad=x_{1}\left(-u_{1 j} d \nu\left(X_{12}\right) \psi+u_{3 j} d \nu\left(X_{23}\right) \psi+u_{4 j} d \nu\left(X_{24}\right) \psi+\cdots+u_{n j} d \nu\left(X_{2 n}\right) \psi\right.
\end{aligned}
$$

This equation simplifies to (24) for $i=1$ and $m=2$. This proves (24) and (23).

From (24) we see that $x_{m}=0$ implies that $\sum_{r=1}^{n} u_{r j} d \nu\left(X_{m r}\right) \psi=0$, for all $j=1, \ldots, d$ so that

$$
x_{m}=0 \Rightarrow \sum_{r=1}^{n} A_{i r}(\sigma) d \nu\left(X_{m r}\right) \psi=0 .
$$

Now by [2], $G(d, n)$ is a finite union of trivial bundles $W_{\alpha}$ with the following local coordinate representation:

$$
\omega_{\alpha}:(\sigma, x) \rightarrow\left(y(\sigma), x_{\alpha}\right) .
$$

Here $\alpha$ is a choice of $n-d$ indices $i_{1}, \ldots, i_{n-d}$ in $\{1, \ldots, n\}, x_{\alpha}=$ $\left(x_{i_{1}}, \ldots, x_{i_{n-d}}\right)$, and $y(\sigma)$ is a local coordinate system for $\sigma \in \pi_{d}\left(W_{\alpha}\right) \subset G_{d, n}$.

To prove that $\Phi_{i} \in \mathscr{E}(G(d, n))$, it suffices to prove that $\left.\Phi_{i}\right|_{W_{\alpha}} \in \mathscr{E}\left(W_{\alpha}\right)$ for each $\alpha$. Suppose first $i$ is one of the indices $i_{1}, \ldots, i_{n-d}$. Since $x_{i}$ is a local coordinate in $W_{\alpha}$, it follows by (25) for $m=i$ that

$$
\Phi_{i}(\sigma, x)=\frac{\sum_{r=1}^{n} A_{i r}(\sigma) d \nu\left(X_{i r}\right) \psi}{x_{i}}
$$

is a $C^{\infty}$ function on $W_{\alpha}$. Suppose next that $i$ is not one of the indices $i_{1}, \ldots, i_{n-d}$. Choose any $m$ in $\left\{i_{1}, \ldots, i_{n-d}\right\}$. Since $x_{m}$ is a local coordinate in $W_{\alpha}$, it follows from (25) that the function

$$
\frac{\sum_{r=1}^{n} A_{i r}(\sigma) d \nu\left(X_{m r}\right) \psi}{x_{m}}
$$

is a $C^{\infty}$ function on $W_{\alpha}$. But by (23), this function equals $\Phi_{i}$. (Strictly speaking, it equals $\Phi_{i}$ at the points $(\sigma, x)$ where $x_{i} \neq 0$, a dense open set in $W_{\alpha}$. But then (26) extends $\Phi_{i}$ to be a $C^{\infty}$ function on all of $W_{\alpha}$.) This proves that $\Phi_{i} \in \mathscr{E}(G(d, n))$ and thus the lemma.

Lemma 4.5. Let the function $F$ be as in Lemma 4.3. Then $F \in \mathscr{S}\left(\mathbb{R}^{n}\right)$.

Proof. By Lemma 4.4, $F \in \mathscr{E}\left(\mathbb{R}^{n}\right)$. We must now prove the estimate

$$
\sup _{x \in R^{n}}\|x\|^{k}\left|\Delta^{m} F(x)\right|<\infty
$$


where $\Delta$ denotes the Laplacian on $\mathbb{R}^{n}$. For any $x \neq 0$ in $\mathbb{R}^{n}$, write $r=\|x\|$ and $x^{\prime}=x / r$. Then $x^{\prime} \in S^{n-1}$ and we have

$$
\Delta=\frac{\partial^{2}}{\partial r^{2}}+\frac{n-1}{r} \frac{\partial}{\partial r}+\frac{1}{r^{2}} L_{x^{\prime}}
$$

where $L_{x^{\prime}}$ represents the Laplace operator on $S^{n-1}$. Then

$$
\|x\|^{k} \cdot \Delta^{m} F(x)=\sum_{\substack{u, v, w \\ \text { finite }}} h_{u v w} r^{u} \frac{\partial^{v}}{\partial r^{v}} L_{x^{\prime}}^{w} F\left(r x^{\prime}\right) .
$$

Since the Casimir operator on $\mathrm{SO}(n)$ projects to the Laplace operator on $S^{n-1}$, we have $L_{x^{\prime}}=c \sum_{i<j} d \lambda\left(X_{i j}^{2}\right)$. Thus each summand in (27) is itself a linear combination of terms of the form

$$
r^{u} \frac{\partial^{v}}{\partial r^{v}} d \lambda\left(X_{1}^{2} \cdots X_{s}^{2}\right) F\left(r x^{\prime}\right)
$$

where each $X_{i}$ is a basis element of $\operatorname{so}(n)$. But since $F(x)=\psi(\sigma, x)$ for $x \in \sigma^{\perp}$ and since, by (19), $d \lambda(U) F(x)=d \nu(U) \psi(\sigma, x)$ for all $U \in \operatorname{so}(n)$, (28) equals

$$
r^{u} \partial^{v} / \partial r^{v} d \nu\left(X_{1}^{2} \cdots X_{s}^{2}\right) \psi\left(\sigma, r x^{\prime}\right)
$$

where $x^{\prime} \in \sigma^{\perp} \cap S^{n-1}$. We will now estimate (29). Let $\Gamma: V \times \mathbb{R}^{n-d} \rightarrow \pi_{d}^{-1}(V)$ be the parametrization of a local trivial subbundle of $G(d, n)$ as in (5), and let $M \subset G_{d, n}$ be a full compact subset of $V$ admitting a local cross section $\eta$ into $O(n)$. We will show that

$$
\sup _{\substack{\sigma \in M \\ x \in R^{n-d}}}\left|r^{u} \frac{\partial^{v}}{\partial r^{v}} d \nu\left(X_{1}^{2} \cdots X_{s}^{2}\right) \psi(\Gamma(\sigma, x))\right|<\infty .
$$

Now

$$
\frac{\partial}{\partial r}=\sum_{d+1}^{n} \frac{x_{i}}{\|x\|} \frac{\partial}{\partial x_{i}}
$$

Also,

$$
\begin{aligned}
& \left(d \nu\left(X_{1}^{2} \cdots X_{s}^{2}\right) \psi\right)(\Gamma(\sigma, x)) \\
& \quad=\left.\frac{\partial^{2 s}}{\partial t_{1}^{2} \cdots \partial t_{s}^{2}} \psi\left(\exp \left(-t_{s} X_{s}\right) \cdots \exp \left(-t_{1} X_{1}\right) \cdot(\sigma, \eta(\sigma) \cdot x)\right)\right|_{t_{i}=0} \\
& \quad=\frac{\partial^{2 s}}{\partial t_{1}^{2} \cdots \partial t_{s}^{2}} \psi\left(\exp \left(-t_{s} X_{s}\right) \cdots \exp \left(-t_{1} X_{1}\right) \cdot \sigma, \exp \left(-t_{s} X_{s}\right) \cdots\right. \\
& \left.\left.\quad \exp \left(-t_{1} X_{1}\right) \cdot \eta(\sigma) \cdot x\right)\right)\left.\right|_{t_{i}=0}
\end{aligned}
$$


But $\exp \left(-t_{s} X_{s}\right) \cdots \exp \left(-t_{1} X_{1}\right) \cdot \eta(\sigma) \cdot x=\eta\left(\exp \left(-t_{s} X_{s}\right) \cdots \exp \left(-t_{1} X_{1}\right) \cdot \sigma\right) \cdot x^{\prime}$ for some $x^{\prime} \in \mathbb{R}^{n-d}$ so

$$
\begin{aligned}
x^{\prime} & =\eta\left(\exp \left(-t_{s} X_{s}\right) \cdots \exp \left(-t_{1} X_{1}\right) \cdot \sigma\right)^{-1} \cdot \exp \left(-t_{s} X_{s}\right) \cdots \exp \left(-t_{1} X_{1}\right) \cdot \eta(\sigma) \cdot x \\
& =k\left(\sigma, t_{1}, \ldots, t_{s}\right) \cdot x,
\end{aligned}
$$

where $k\left(\sigma, t_{1} \ldots, t_{s}\right) \in O(n-d)$ is a $C^{\infty}$ function of $\sigma$ and $t_{1} \ldots, t_{s}$ such that $k(\sigma, 0, \ldots, 0)=I_{n-d}$, the $(n-d) \times(n-d)$ identity matrix. Thus,

$$
\begin{aligned}
d \nu( & \left.X_{1}^{2} \cdots X_{s}^{2}\right) \psi(\Gamma(\sigma, x)) \\
& =\left.\frac{\partial^{2 s}}{\partial t_{1}^{2} \cdots \partial t_{s}^{2}}(\psi \circ \Gamma)\left(\exp \left(-t_{s} X_{s}\right) \cdots \exp \left(-t_{1} X_{1}\right) \cdot \sigma, k(\sigma, t) \cdot x\right)\right|_{t_{i}=0} \\
& =\sum_{\beta, E} C_{\beta, E} x^{\beta} E_{\sigma}(\psi \circ \Gamma)(\sigma, x)
\end{aligned}
$$

where the sum runs through a finite set of multi-indices $\beta=\left(\beta_{d+1}, \ldots, \beta_{n}\right)$ and differential operators $E$ on $G_{d, n}$. Applying

$$
r^{u} \frac{\partial^{v}}{\partial r^{v}}=\|x\|^{u}\left(\sum_{d+1}^{n} \frac{x_{i}}{\|x\|} \frac{\partial}{\partial x_{i}}\right)^{v}
$$

to the above sum yields a finite linear combination of terms of the form $x^{\beta^{\prime}}\|x\|^{\kappa} D_{x} E_{\sigma}(\psi \circ \Gamma)(\sigma, x)$. Here $\kappa$ may be a negative exponent but this does not matter. Since $\psi \in \mathscr{S}(G(d, n))$, each of the above terms is bounded for all $(\sigma, x) \in M \times \mathbb{R}^{n-d}$, with $\|x\| \geq 1$, say. This shows that (30), and hence (28) and (27) are bounded.

Theorem 4.6. $\mathscr{S}\left(\mathbb{R}^{n}\right)^{-}=\mathscr{S}_{D}(G(d, n))$.

Proof. We have already seen that $\mathscr{D}\left(\mathbb{R}^{n}\right)^{\wedge} \subset \mathscr{S}_{D}(G(d, n))$. So let $\varphi \in$ $\mathscr{S}_{D}(G(d, n))$, let $\psi=\tilde{\varphi}$, and let $F$ be as in Lemma 4.3. By lemma 4.5, $F \in \mathscr{S}\left(\mathbb{R}^{n}\right)$. By projection-slice, the partial Fourier transform $(\hat{f})^{\sim}$ satisfies

$$
(\hat{f})^{\sim}(\sigma, x)=\tilde{f}(x)=F(x)=\tilde{\varphi}(\sigma, x) .
$$

Taking inverse partial Fourier transform, we obtain $\hat{f}=\varphi$, as desired.

\section{THE RANGE OF THE DUAL $d$-PLANE TRANSFORM}

Consider the dual $d$-plane transform $\varphi \rightarrow \check{\varphi}$ from functions on $G(d, n)$ to functions on $\mathbb{R}^{n}$, given by

$$
\check{\varphi}(x)=\int_{G_{d, n}} \varphi(\tau(x) \sigma) d \sigma, \quad \varphi \in \mathscr{E}(G(d, n)),
$$

where $d \sigma$ is the normalized $O(n)$-invariant measure on $G_{d, n}=O(n) / O(d) \times$ $O(n-d)$. In this section we will prove the following theorem. 
Theorem 5.1. $\mathscr{E}(G(d, n))^{\nu}=\mathscr{E}\left(\mathbb{R}^{n}\right)$.

This was proven by Hertle [13] in the case $d=n-1$.

Before proceeding with the proof, let us first gather some preliminary facts. Since $G(d, n)=E(n) / E(d) \times O(n-d)$ is a quotient of unimodular groups, there exists a unique (up to constant multiple) $C^{\infty}$ measure $\mu$ on $G(d, n)$ which is invariant under $\tau(E(n))$. We fix the measure $\mu$ to satisfy

$$
\int_{G(d, n)} \varphi(\xi) d \mu(\xi)=\int_{G_{d, n}} \int_{\sigma^{\perp}} \varphi(\sigma, x) d \sigma^{\perp}(x) d \sigma, \quad \varphi \in \mathscr{D}(G(d, n)) .
$$

Then by general principles [11],

$$
\int_{G(d, n)} \hat{f}(\xi) \varphi(\xi) d \mu(\xi)=\int_{\mathbb{R}^{n}} f(x) \check{\varphi}(x) d x
$$

for all $f \in \mathscr{D}\left(\mathbb{R}^{n}\right), \varphi \in \mathscr{E}(G(d, n))$. If $D$ is a differential operator on $G(d, n))$, the adjoint operator with respect to $\mu$ will be denoted $D^{*}$. Since $\mu$ is preserved under $\tau(E(n))$, we have $d \nu(X)^{*}=-d \nu(X)$ for all $X \in \mathfrak{e}$. From this one can easily see that $d \nu\left(V_{i j l}\right)^{*}=d \nu\left(V_{i j l}\right)$ for all $i, j, l$.

By [12], the map $f \rightarrow \hat{f}$ is continuous from $\mathscr{D}\left(\mathbb{R}^{n}\right)$ into $\mathscr{D}(G(d, n))$ and the map $\varphi \rightarrow \check{\varphi}$ is continuous from $\mathscr{E}(G(d, n))$ to $\mathscr{E}\left(\mathbb{R}^{n}\right)$. Using the relation (31), these maps have natural extensions to continuous maps $S \rightarrow \widehat{S}$ from $\mathscr{E}^{\prime}\left(\mathbb{R}^{n}\right)$ into $\mathscr{E}^{\prime}(G(d, n))$ and $T \rightarrow \check{T}$ from $\mathscr{D}^{\prime}(G(d, n))$ to $\mathscr{D}^{\prime}\left(\mathbb{R}^{n}\right)$ [10].

If $\gamma \in \mathbb{C}_{n}=\mathbb{C}-\{n, n+2, n+4, \ldots\}, I^{\gamma}$ will denote the Riesz potential

$$
I^{\gamma} f(x)=\frac{1}{H_{n}(\gamma)} \int_{\mathbb{R}^{n}} f(y)\|x-y\|^{\gamma-n} d y,
$$

for $f \in \mathscr{S}\left(\mathbb{R}^{n}\right)$, where $H_{n}(\gamma)=2^{\gamma} \pi^{n / 2} \Gamma\left(\frac{1}{2} \gamma\right) \Gamma\left(\frac{1}{2}(n-\gamma)\right)^{-1}$. When $\operatorname{Re}(\gamma) \leq$ 0 , this is interpreted as analytic continuation. For any real number $p$ such that $-2 p \in \mathbb{C}_{n}$, the fractional power $\Delta^{p}$ of the Laplacian $\Delta$ on $\mathbb{R}^{n}$ is then defined by $(-\Delta)^{p} f=I^{-2 p} f\left(f \in \mathscr{S}\left(\mathbb{R}^{n}\right)\right)$. The Fourier transform $\tilde{f}$ of $f$ satisfies $\left((-\Delta)^{p} f\right)^{\sim}(u)=\|u\|^{2 p} \tilde{f}(u)$.

According to Ortner [15, Satz 9], the following formula holds: if $\gamma, \mu \in \mathbb{C}_{n}$ such that $\operatorname{Re}(\gamma+\mu)<n$, then

$$
I^{\gamma} I^{\mu} f=I^{\gamma+\mu} f
$$

for all $f \in \mathscr{S}\left(\mathbb{R}^{n}\right)$. Suppose now $\varphi \in \mathscr{S}(G(d, n))$. In accordance with (32), the fractional power $\square^{k} \varphi$ is defined by

$$
\begin{aligned}
(-\square)^{k} \varphi(\sigma, x) & =I^{-2 k} \varphi(\sigma, x) \\
& =H_{n-d}(-2 k)^{-1} \int_{\sigma^{\perp}} \varphi(\sigma, y)\|x-y\|^{-2 k-(n-d)} d \sigma^{\perp}(y)
\end{aligned}
$$

interpreted, as above, for $k \geq 0$ by analytic continuation. Using the parametrization (5) of $G(d, n)$, we have

$$
(-\square)^{k} \varphi(\Gamma(\sigma, x))=H_{n-d}(-2 k)^{-1} \int_{\mathbb{R}^{n-d}} \varphi \circ \Gamma(\sigma, u)\|x-u\|^{-2 k-(n-d)} d u .
$$


Thus, under the parametrization $\Gamma$, the operator $(-\square)^{k}$ is given by convolution with a tempered distribution in $\mathbb{R}^{n-d}$. Hence $(-\square)^{k} \varphi \in \mathscr{E}(G(d, n))$ and the map $\varphi \rightarrow \check{\varphi}$ is continuous from $\mathscr{S}(G(d, n))$ into $\mathscr{E}(G(d, n))$.

Lemma 5.2. Let $\varphi \in \mathscr{S}(G(d, n))$ and $k \geq 0$. Then there exists a constant $C$ such that $\left|(-\square)^{k} \varphi(\sigma, u)\right| \leq C(1+\|u\|)^{-2 k-(n-d)}$ for all $\sigma \in G_{d, n}, u \in \sigma^{\perp}$.

Proof. This follows directly from Lemma 1 of [15], applied to each local parametrization $\Gamma$ of $G(d, n)$, since the set of functions $\{x \rightarrow \varphi(\Gamma(\sigma, x)) \mid \sigma \in V\}$ is a bounded subset (in the topology) of $\mathscr{S}\left(\mathbb{R}^{n-d}\right)$.

By Lemma 5.2, the partial Fourier transform $\left((-\square)^{k} \varphi\right)^{\sim}$ is given by an absolutely convergent integral for $\varphi \in \mathscr{S}(G(d, n))$ and satisfies

$$
\left((-\square)^{k} \varphi\right)^{\sim}(\sigma, u)=\|u\|^{2 k} \tilde{\varphi}(\sigma, u)
$$

Lemma 5.3. Let $f \in \mathscr{S}\left(\mathbb{R}^{n}\right)$. Then for any $k \geq 0$,

$$
\left((-\Delta)^{k} f\right)^{\wedge}=(-\square)^{k} \hat{f}
$$

Proof. If $k \in \mathbb{Z}^{+},(35)$ is obvious, so we assume $k \notin \mathbb{Z}^{+}$. By Lemma 1 of [15], $(-\Delta)^{k} f(x)=O\left(\|x\|^{-n-2 k}\right)$ as $\|x\| \rightarrow \infty$ so $\left((-\Delta)^{k} f\right)^{-}$is well-defined. (35) follows, since the partial Fourier transform of both sides is $\|u\|^{2 k} \tilde{f}(u)$.

By (33) and (35) we have the following inversion formula for the transform $f \rightarrow \hat{f}$ (see [10]):

$$
c_{n} f=\left(\left((-\Delta)^{d / 2} f\right)^{\wedge}\right)^{\natural}=\left((-\square)^{d / 2} \hat{f}\right)^{\varsigma}
$$

where $c_{n}=4 \pi^{d / 2} \Gamma\left(\frac{1}{2} n\right) \Gamma\left(\frac{1}{2}(n-d)\right)^{-1}$.

Now it is easy to see that if $k \geq 0$,

$$
\int_{G(d, n)}(-\square)^{k} \varphi(\xi) \cdot \psi(\xi) d \mu(\xi)=\int_{G(d, n)} \varphi(\xi) \cdot(-\square)^{k} \psi(\xi) d \mu(\xi)
$$

for all $\varphi, \psi \in \mathscr{D}(G(d, n))$. Thus for any $T \in \mathscr{E}^{\prime}(G(d, n))$, we can define the distribution $(-\square)^{k} T \in \mathscr{D}^{\prime}(G(d, n))$ by the formula $\left((-\square)^{k} T\right)(\varphi)=$ $T\left((-\square)^{k} \varphi\right)$, for any $\varphi \in \mathscr{D}(G(d, n))$. Since the map $\varphi \rightarrow(-\square)^{k} \varphi$ is continuous from $\mathscr{D}(G(d, n))$ into $\mathscr{E}(G(d, n))$, the adjoint map $T \rightarrow(-\square)^{k} T$ is continuous from $\mathscr{E}^{\prime}(G(d, n))$ to $\mathscr{D}^{\prime}(G(d, n))$.

Lemma 5.4. We have

(i) $\left((-\square)^{k} \varphi\right)^{\tau(g)}=(-\square)^{k} \varphi^{\tau(g)}$,

(ii) $\left((-\square)^{k} T\right)^{\tau(g)}=(-\square)^{k} T^{\tau(g)}$,

for all $g \in E(n), \varphi \in \mathscr{D}(G(d, n)), T \in \mathscr{E}^{\prime}(G(d, n))$.

Proof. Upon taking the partial Fourier transform of both sides, (i) follows from (34) and (10) and (11) in Lemma 4.2. (ii) follows by applying both sides to a given $\varphi \in \mathscr{D}$ and using (i).

Now Theorem 5.1 is a consequence of the following two lemmas. 
Lemma 5.5 (Helgason [12]). Let $G / K$ and $G / H$ be homogeneous spaces in duality, $K$ compact. Let $N$ denote the kernel of $S \rightarrow \widehat{S}$ on $\mathscr{E}^{\prime}(G / K)$. Assume the subspace $\mathscr{E}^{\prime}(G / K)^{\wedge} \subset \mathscr{E}^{\prime}(G / H)$ is closed, $\mathscr{E}^{\prime}(G / H)$ carrying the strong topology. Then

$$
\mathscr{E}(G / H)^{\swarrow}=N^{\perp},
$$

where $N^{\perp}=\{f \in \mathscr{E}(G / K) \mid S(f)=0$ for all $S \in N\}$.

A special case of Lemma 5.5 was first proven by Hertle [13]. In the present situation $G / K=E(n) / O(n)=\mathbb{R}^{n}$ and $G / H=E(n) / E(d) \times O(n-d)=$ $G(d, n)$.

Lemma 5.6. $\mathscr{E}^{\prime}\left(\mathbb{R}^{n}\right)^{\wedge}=\left\{T \in \mathscr{E}^{\prime}(G(d, n)) \mid d \nu\left(V_{i j l}\right) T=0\right.$ for all $\left.i, j, l\right\}$.

Assuming the above two lemmas, Theorem 5.1 is proven as follows. By Lemma 5.6, $\mathscr{E}^{\prime}\left(\mathbb{R}^{n}\right)^{\wedge}$ is weakly closed, hence strongly closed in $\mathscr{E}^{\prime}(G(d, n))$. By the injectivity of the map $S \rightarrow \widehat{S}$ [10], we have $N=0$, so Theorem 5.1 follows from (38).

Proof of Lemma 5.6. Lemma 5.6 is obtained from Theorem 4.6 by an approximation argument. We proceed as follows. If $\phi \in \mathscr{D}(E(n))$ and $\Psi$ is a distribution on $\mathbb{R}^{n}$ or $G(d, n)$, we write $\phi * \Psi=\int_{E(n)} \phi(g) \cdot \Psi^{\tau(g)} d g$, where $d g$ is the Haar measure on $E(n)$. Then $\phi * \Psi$ is a $C^{\infty}$ function [11], compactly supported if $\Psi$ is.

Now let $\left\{\phi_{m}\right\}_{m=1}^{\infty} \subset \mathscr{D}(E(n))$ be any sequence converging in $\mathscr{E}^{\prime}(E(n))$ to $\delta_{e}$, the $\delta$-function at the identity of $E(n)$. If $S \in \mathscr{E}^{\prime}\left(\mathbb{R}^{n}\right)$, then

$$
\lim _{m \rightarrow \infty} \phi_{m} * S=S
$$

in the (strong) topology of $\mathscr{E}^{\prime}\left(\mathbb{R}^{n}\right)$, so that

$$
\lim _{m \rightarrow \infty}\left(\phi_{m} * S\right)^{\wedge}=\widehat{S}
$$

in $\mathscr{E}^{\prime}(G(d, n))$. Thus,

$$
d \nu\left(V_{i j l}\right) \widehat{S}=\lim _{m \rightarrow \infty} d \nu\left(V_{i j l}\right)\left(\phi_{m} * S\right)^{\wedge}=0
$$

by Theorem 4.6. On the other hand, let $T \in \mathscr{C}^{\prime}(G(d, n))$ satisfy $d \nu\left(V_{i j l}\right) T=0$ for all $i, j, l$. We have $\lim _{m \rightarrow \infty} \phi_{m} * T=T$ in $\mathscr{E}^{\prime}(G(d, n))$ and

$$
\begin{aligned}
d \nu\left(V_{i j l}\right)\left(\phi_{m} * T\right) & =\int_{E(n)} \phi_{m}(g) d \nu\left(V_{i j l}\right)\left(T^{\tau(g)}\right) d g \\
& =\int_{E(n)} \phi_{m}(g)\left(\left(d \nu \operatorname{Ad}\left(g^{-1}\right) V_{i j l}\right) T\right)^{\tau(g)} d g
\end{aligned}
$$

By Lemma 4.1, $\operatorname{Ad}\left(g^{-1}\right) V_{i j l}=\sum c_{u s r} V_{u s r}$, so by the hypothesis on $T$, the right-hand side equals zero. Thus, by Theorem 4.6 and the Support Theorem 
[9], $\phi_{m} * T=\hat{f}_{m}$, for some $f_{m} \in \mathscr{D}\left(\mathbb{R}^{n}\right)$. From the inversion formula (36), we have

$$
c_{n} f_{m}=\left((-\square)^{d / 2}\left(\phi_{m} * T\right)\right)^{2} .
$$

Now by Lemma 5.3, $(-\square)^{d / 2}\left(\phi_{m} * T\right)=\phi_{m} *(-\square)^{d / 2} T$, which converges to $(-\square)^{d / 2} T$ in $\mathscr{D}^{\prime}(G(d, n))$. Thus, $f_{m}=c_{n}^{-1}\left(\phi_{m} *(-\square)^{d / 2} T\right)^{\nu}$ converges to the distribution $\left.S=\left((-\square)^{d / 2} T\right)\right)^{\sim}$ in $\mathscr{D}^{\prime}\left(\mathbb{R}^{n}\right)$. But the functions $\phi_{m} * T$ are all supported on a common compact subset of $G(d, n)$, so by the Support Theorem, the functions $f_{m}$ are all supported in a common subset of $\mathbb{R}^{n}$. Thus $S \in \mathscr{E}^{\prime}\left(\mathbb{R}^{n}\right)$ and $\left\{f_{m}\right\}$ converges to $S$ in the space $\mathscr{E}^{\prime}\left(\mathbb{R}^{n}\right)$. It follows that

$$
T=\lim _{m \rightarrow \infty} \phi_{m} * T=\lim _{m \rightarrow \infty} \hat{f}_{m}=\hat{S},
$$

the convergence being in $\mathscr{E}^{\prime}(G(d, n))$. This proves Lemma 5.6.

The proof of Theorem 5.1 is now complete.

Remark. For $d<n-1$, it is difficult to formulate a range theorem for $\mathscr{E}^{\prime}\left(\mathbb{R}^{n}\right)^{\wedge}$ in terms of moment conditions. The reason is that $\sigma$ and $x \in \sigma^{\perp}$ are not independent parameters.

Let $H$ be the nullspace of the transform $\varphi \rightarrow \check{\varphi}$ on $\mathscr{E}(G(d, n))$, i.e., the annihilator of $\mathscr{E}^{\prime}\left(\mathbb{R}^{n}\right)^{\wedge}$. Since the operators $d \nu\left(V_{i j l}\right)$ are all selfadjoint, Theorem 5.1 implies that $H$ is the double annihilator, that is to say the weak closure, of the subspace $\sum_{i<j<1} d \nu\left(V_{i j l}\right) \mathscr{E}(G(d, n))$. In particular, $H$ contains functions of compact support. By contrast, the dual transform is injective on $\mathscr{D}(G(n-1, n))[4,18]$. For $d=1$ and $n=3, H$ is thus the weak closure of $d \nu\left(V_{123}\right) \mathscr{E}(G(1,3)) \subset \mathscr{E}(G(1,3))$. It is an interesting problem to determine whether $H=d \nu\left(V_{123} \mathscr{E}(G(1,3))\right.$, i.e., whether or not there are functions in $H$ that do not belong to the range of the operator $d \nu\left(V_{123}\right)$.

\section{REFERENCES}

1. I. M. Gelfand, I. M. Graev, and S. J. Shapiro, Differential forms and integral geometry, Functional Anal. Appl. 3 (1969), 24-40.

2. I. M. Gelfand, S. G. Gindikin, and M. I. Graev, Integral geometry in affine and projective spaces, J. Soviet Math. 18, No. 3 (1982), 39-164. (Translated from Itogi Nauki i Tekhniki, Seriya Sovremennye Problemy Matematiki 16 (1980), 53-226.)

3. F. Gonzalez, Ph.D. Thesis, MIT, 1984.

4. __ Radon transforms on Grassmann manifolds, J. Funct. Anal. 71 (1987), 229-362.

5. __ Bi-invariant differential operators on the Euclidean motion group and applications to generalized Radon transforms, Ark. Mat. 26 (1988), 191-204.

6. F. Gonzalez and S. Helgason, Invariant differential operators on Grassmann manifolds, Adv. in Math. 60 (1986), 81-91.

7. E. Grinberg, Euclidean Radon transforms: ranges and restrictions, Contemp. Math. 63 (1987), 109-134.

8. S. Helgason, Differential operators on homogeneous spaces, Acta Math. 102 (1959), 239299. 
9. ___ The Radon transform on Euclidean spaces, compact two-point homogeneous spaces, and Grassman manifolds, Acta Math. 113 (1965), 153-180.

10. __ The Radon transform, Birkhäuser, Basel and Boston, Mass., 1980.

11. __ Groups and geometric analysis, Academic Press, Orlando, Fla., 1984.

12. _ - Some results on Radon transforms, Huygen's principle, and x-ray transforms, Contemp. Math. 63 (1987), 151-178.

13. A. Hertle, On the range of the Radon transform and its dual, Math. Ann. 267 (1984), 91-99.

14. F. John, The ultrahyperbolic differential equation with four independent variables, Duke Math. J. 4 (1938), 300-322.

15. N. Ortner, Faltung hypersingulärer Integraloperatoren, Math. Ann. 298 (1980), 19-46.

16. F. Richter, Differentialoperatoren auf Euklidischen $k$-Ebenräumen and Radon-Transformation, Dissertation, Humboldt-Universität zu Berlin, 1986.

17. D. Solmon, The x-ray transform, J. Math. Anal. Appl. 56 (1976), 61-83.

18. _ Asymptotic properties for the dual Radon transform and applications, Math. Z. 195 (1987), 321-343.

19. N. Wallach, Harmonic analysis on homogeneous spaces, Dekker, New York, 1973.

Department of Mathematics, Tufts University, Medford, Massachusetts 02155 